\title{
Disfunciones cognitivas en adultos mayores con depresión
}

\author{
Jorge Darío López Sánchez y Dora Elizabeth Granados Ramos
}

\begin{abstract}
Resumen
La depresión es uno de los trastornos mentales con mayor prevalencia a nivel mundial. Quien la padece ve afectada su funcionalidad diaria debido a la sintomatología negativa, aislamiento social y disminución de la actividad psicomotora. Si se presenta durante el envejecimiento, puede incapacitar a la persona cuando, además de los síntomas depresivos, existen alteraciones cognitivas significativas. El tratamiento usual para los trastornos depresivos son los Inhibidores Selectivos de la Recaptación de Serotonina (ISRSS), no obstante, en los adultos mayores que presentan disfunción ejecutiva, dicho tratamiento puede tener menor efectividad, por lo que se deben analizar otros fármacos y métodos psicoterapéuticos que mejoren la calidad de vida de las personas. Por lo anterior, el objetivo de este artículo fue proporcionar una descripción de las disfunciones cognitivas presentes en adultos mayores con depresión, así como brindar algunas pautas para su tratamiento.
\end{abstract}

Palabras clave: funciones ejecutivas, depresión, cognición, terapia.

\section{COGNITIVE DYSFUNCTIONS IN OLDER ADULTS WITH DEPRESSION}

\begin{abstract}
Depression is one of the most prevalent mental disorders worldwide. The daily functionality of those who suffer from it is affected due to negative symptoms, social isolation, and decreased psychomotor activity. If it occurs in an elderly person, it can be incapacitating when, in addition to depressive symptoms, it is accompanied by cognitive disorders. The usual treatment for depressive disorders is Selective Serotonin Reuptake Inhibitors (SSRIS), however, in elderly adults with executive dysfunction, this treatment may be less effective, thus other psychotherapeutic drugs and methods should be analyzed to improve people's quality of life. Therefore, the objective of this article is to provide a description of the cognitive dysfunctions present in older adults with depression, as well as some guidelines for their treatment.
\end{abstract}

Keywords: executive function, depression, cognition, therapy.

Recepción: 08/06/2020. Aprobación: 18/01/2021. Dol: http://doi.org/10.22201/cuaieed.16076079e.2021.22.2.2 


\section{Jorge Darío López Sánchez}

$$
\begin{array}{r}
\text { jorgelopez02@uv.mx } \\
\text { orcid.org/0000-0002-7912-3593 } \\
\text { http://cienciaycerebro.com/ }
\end{array}
$$

Licenciado en Psicología por la Universidad Veracruzana. Maestro en Neuroetología y Especialista en Métodos Estadísticos por la misma institución. Sus líneas de investigación son la neurofisiología y neuropsicología del envejecimiento. Actualmente es estudiante del Doctorado en Neuroetología por el Instituto de Neuroetología de la Universidad Veracruzana. También es docente de la Facultad de Psicología Región Xalapa y colaborador del Laboratorio de Psicobiología, donde desarrolla investigaciones sobre los procesos neurofisiológicos y neuropsicológicos del envejecimiento, así como sobre los procesos cognitivos en adultos.

\section{Dora Elizabeth Granados Ramos}

dgranados@uv.mx orcid.org/0000-0002-7019-6745

Doctora en Neurociencias por la Universidad de Guadalajara en 2003, Maestra en Rehabilitación Neurológica por la UAm Xochimilco en 1993 y Licenciada en Educación Especial por la Escuela Normal de Especialización en 1989. Su línea de investigación es el neurodesarrollo del lenguaje y pensamiento. Actualmente es Profesora Investigadora Titular C, adscrita al Laboratorio de Psicobiología de la Facultad de Psicología Región Xalapa, donde desarrolla investigaciones sobre la evaluación neuropsicológica de pacientes con dificultades de aprendizaje. Es integrante del Sistema Nacional de Investigadores Nivel 1. 


\section{Introducción}

La depresión es un trastorno mental común que se caracteriza por un estado anímico alterado, en el que el enfermo presenta tristeza, anhedonia, ${ }^{1}$ sentimientos de culpa e inferioridad; también se ve afectado el apetito, el sueño, y la capacidad cognitiva (American Psychological Association [APA], 2020). En 2015, se estimó que más de 300 millones de personas padecen este trastorno y se considera que es una de las principales causas de discapacidad, a diferencia de la tristeza, el miedo o alguna otra emoción exacerbada. La depresión es una enfermedad que puede ser diagnosticada clínicamente y tratada con fármacos antidepresivos y psicoterapia. El riesgo de padecerla se incrementa por condiciones de pobreza, enfermedad, abuso de sustancias, desempleo, o por eventos de la vida adversos como el fallecimiento de algún familiar y la insatisfacción profesional (ver figura 1). Las mujeres tienen mayor prevalencia de depresión que los hombres (5.1\% contra 3.6\% a nivel mundial). Asimismo, las muertes por suicidio derivadas de una depresión se calculan en alrededor de 800000 personas al año, por lo que es uno de los trastornos que los organismos de salud pública buscan prevenir e intervenir (World Health Orgnization [wHo], 2017; 2019).

Figura 1. Factores y síntomas asociados a la depresión en el envejecimiento. Elaboración propia basada en: WHO, 2017; APA, 2020.

\footnotetext{
1 Incapacidad para experimentar placer en actividades que normalmente son agradables
} para la persona.

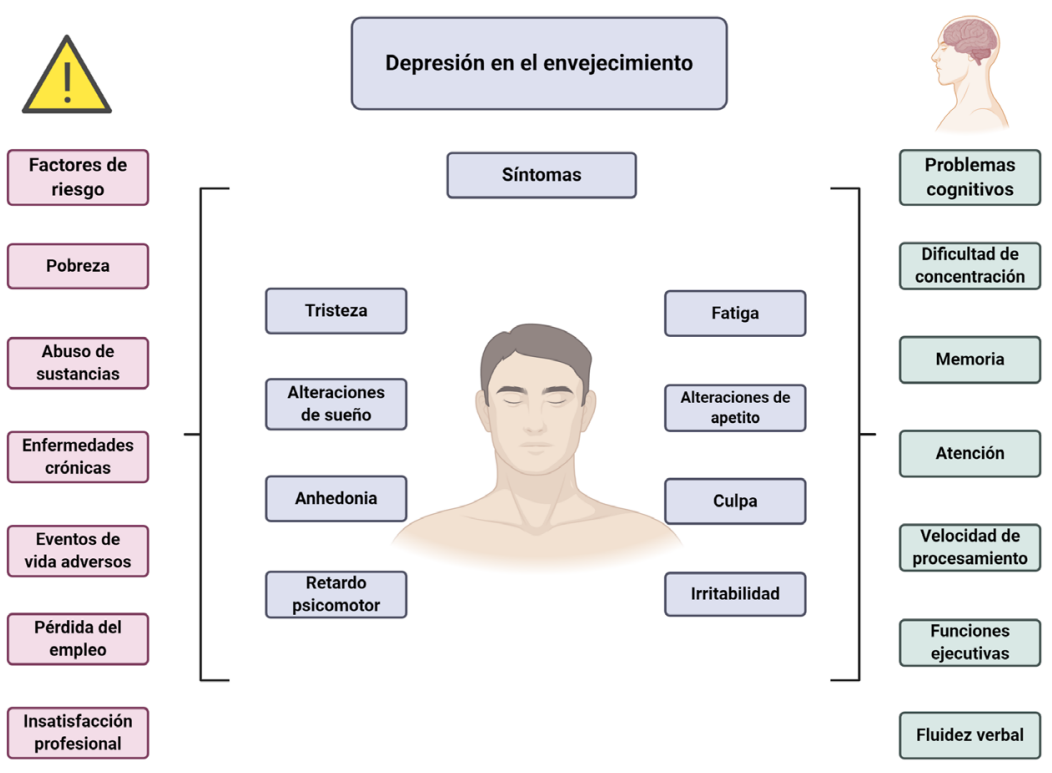

En reportes derivados de datos provenientes del Institute for Health Metrics and Evaluation (Ritchie y Roser, 2018), se describe la depresión como el segundo trastorno de salud mental de mayor prevalencia, con 3.4\% a nivel mundial, únicamente sobrepasado por los trastornos de ansiedad cuya prevalencia es de 3.8\%. En México, la prevalencia de la depresión es de 2.8\%, que corresponde a 3.3 millones de mexicanos. Ésta incrementa en función de la edad, duplicándose a partir de los 50 años (6\% de 50 a 69 años y 6.2\% para los mayores de 70 años), de modo que es más frecuente en la población envejecida (Ritchie y Roser, 2018). Si el episodio depresivo se presenta por primera vez en este 
último rango de edad, se diagnostica como depresión tardía (late life depression) $y$, de no tratarse, puede incrementar la mortalidad al disminuir el autocuidado ante enfermedades crónicas como la diabetes o la hipertensión, y al aumentar la probabilidad de desarrollar demencia (Fischer et al., 2008; Weisenbach, Boore, y Kales, 2012; Brown et al., 2014; Ritchie y Roser, 2018).

\section{Procesos cognitivos afectados en la depresión}

Los trastornos depresivos, además de modificar el estado anímico, también afectan la forma en que la persona percibe los estímulos de su entorno: disminuyen las respuestas conductuales y cognitivas, se modifican negativamente los significados que se asignan a las experiencias personales y se deteriora el desempeño de procesos psicológicos como la memoria y la atención (Bogner et al., 2007; Donovan et al., 2017; Pantzar et al., 2017; Baune et al., 2018).

Las complicaciones cognitivas presentes en la depresión deben considerarse a profundidad, dado que dichas complicaciones van a decrementar la capacidad personal para interactuar socialmente, continuar actividades laborales, y apegarse a tratamientos farmacológicos y psicoterapéuticos (Pantzar et al., 2017; Weisenbach, Boore y Kales, 2012; Baune et al., 2018).

En adultos mayores, los procesos cognitivos más afectados en personas con depresión son la memoria, la atención, la velocidad de procesamiento de la información, las funciones ejecutivas y la fluidez verbal (Fischer et al., 2008; Crocco, Castro y Loewenstein, 2010; Weisenbach, Boore y Kales, 2012; Pantzar et al., 2017; Thomas y O'Brien, 2008). No obstante, dichos déficits pueden aminorarse después del tratamiento, a esto se le denomina reversibilidad del síntoma. Debido a ello, algunos investigadores consideran que los déficits en los procesos cognitivos, a excepción de los de velocidad de procesamiento, son un estado transitorio asociado con la depresión y no un rasgo característico del trastorno, de ahí la importancia de atenderlos (Pantzar et al., 2017).

Se han descrito tres subtipos de disfuncionalidad cognitiva, asociadas con depresión, en adultos mayores. El primer subtipo se caracteriza por presentar únicamente complicaciones en la memoria. Los otros dos presentan combinaciones de dificultades de memoria y funciones ejecutivas o de memoria y atención. Sin embargo, quienes presentan disfunciones ejecutivas tienden a ser los de mayor alteración funcional y, aún cuando la sintomatología depresiva disminuye, estos adultos mayores mantienen algunas complicaciones cognitivas, por lo que la identificación temprana permite implementar estrategias terapéuticas más adecuadas para ellos (Crocco, Castro y Loewenstein, 2010; Weisenbach, Boore y Kales, 2012).

De los procesos mencionados, la disfunción ejecutiva suele agravar la calidad de vida de las personas, esto se debe a que las funciones ejecutivas son responsables de la planificación conductual, la secuenciación de actividades 
"Disfunciones cognitivas en adultos mayores con depresión" Jorge Darío López Sánchez y Dora Elizabeth Granados Ramos

Vol. 22, Núm. 2, marzo-abril 2021

Revista Digital Universitaria

y pensamientos, la abstracción y la integración emocional. Por esta razón, su deterioro afecta la capacidad de realizar las actividades de la vida diaria (AVD), tales como bañarse, vestirse, alimentarse, movilizarse, usar el sanitario, y también impacta en las actividades instrumentales de la vida diaria (AIvD), entre las que se incluyen el cuidado de otras personas o mascotas, uso de dinero, cuidado del hogar, preparación de los alimentos, o la adquisición de productos diversos (Alexopoulos, 2001; Weisenbach, Boore y Kales, 2012). Por otra parte, la velocidad de procesamiento (también afectada en la disfunción ejecutiva) permite mantener y manipular información para su uso posterior. Si dicha velocidad decrementa, afectará directamente el desempeño de los procesos de atención y memoria, por lo tanto, el adulto mayor tendrá dificultad para realizar las actividades que dependan de ellos (Thomas y O'Brien, 2008).

En las disfunciones ejecutivas existe un desempeño disminuido en tareas en las que se debe inhibir la respuesta, como la tarea stroop. En ella, se le presenta al evaluado nombres de colores (verde, rojo, azul o amarillo), impresos en tinta de un color que no concuerda con su nombre (palabra verde impresa en tinta azul), por lo que la persona debe leer las palabras e inhibir una de las dos características (generalmente el nombre) para responder a lo que el evaluador solicita. El desempeño disminuido en ésta y en otras pruebas enfocadas en la planeación conductual se ha asociado con efectos retardados del tratamiento farmacológico y a incrementos en la probabilidad de presentar otro episodio depresivo. Esto se debe a que dichos síntomas son característicos de la depresión isquémica subcortical, también llamada depresión vascular, condición que resulta en el endurecimiento de los vasos sanguíneos, lo que, en consecuencia, impide el flujo normal de la sangre al cerebro, afectando principalmente estructuras subcorticales y de proyecciones hacia la corteza prefrontal, que se encarga del procesamiento ejecutivo (Alexopoulos, 2001; Bogner, et al., 2007; Brown et al., 2014).

Se ha propuesto un modelo para explicar los déficits ejecutivos presentes en los trastornos depresivos, que se ha denominado disfunción ejecutiva depresiva, traducido de su nombre en inglés: depression-executive dysfunction (DED). Los que padecen DED presentan, además de alteraciones en funciones ejecutivas, retraso psicomotor, sintomatología depresiva limitada y dificultad para realizar actividades diarias (Alexopoulos, 2001).

Dicho modelo busca predecir la respuesta de las personas al tratamiento de la depresión con fármacos antidepresivos y considera que la sintomatología depresiva puede ser resultado de anormalidades neuroanatómicas en los circuitos fronto-límbico y fronto-estriado (Alexopoulos, 2001), que son responsables, entre otras funciones, de la regulación del estado anímico y del funcionamiento ejecutivo (ver figura 2). Además, las explicaciones de este modelo concuerdan con otras condiciones como la depresión vascular, descrita previamente (McLennan y Mathias, 2010). 
Figura 2. Interacción entre estructuras subcorticales y la corteza prefrontal. Elaboración propia basada en: Alexopoulos, 2001.

Figura 3. Mecanismo de acción de los Inhibidores Selectivos de la Recaptación de Serotonina.

Elaboración propia basada en: Hiemke y Härtter, 2000.

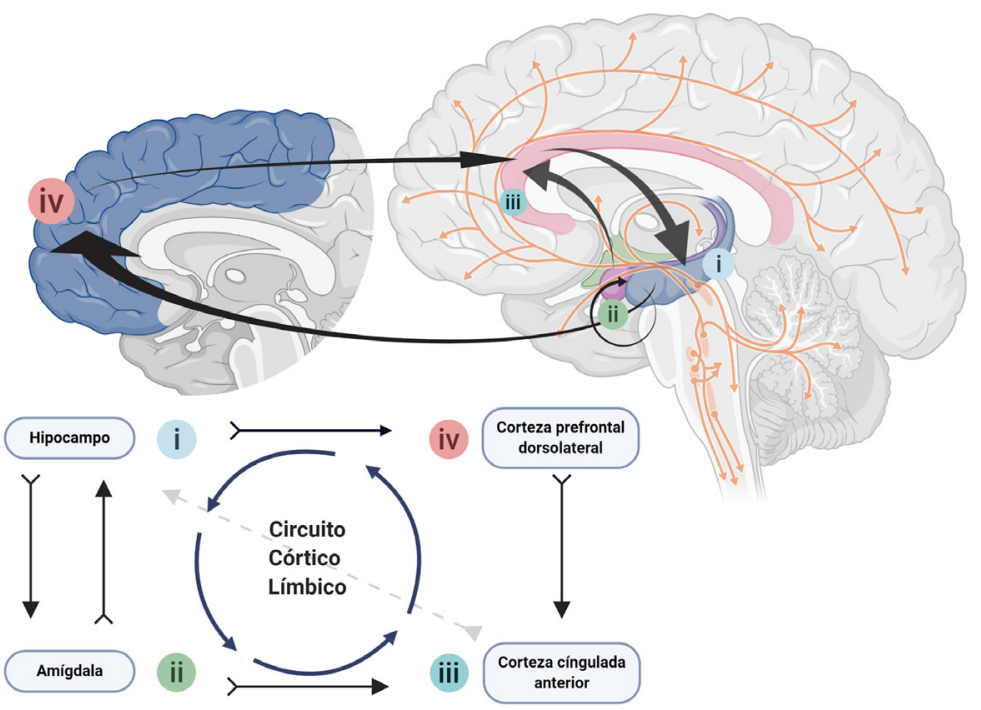

Actualmente, el abordaje farmacológico principal para el trastorno depresivo consiste en el tratamiento con Inhibidores Selectivos de la Recaptación de Serotonina (ISRS), que, al bloquear el proceso recaptación de serotonina (5-HT) hacia la neurona presináptica, incrementan la cantidad de este neurotransmisor en la hendidura sináptica, favoreciendo así su disponibilidad para los receptores de la neurona postsináptica (ver figura 3). No obstante, los isRs tienen poco o nulo efecto sobre la concentración de otros neurotransmisores (Hiemke y Härtter, 2000).

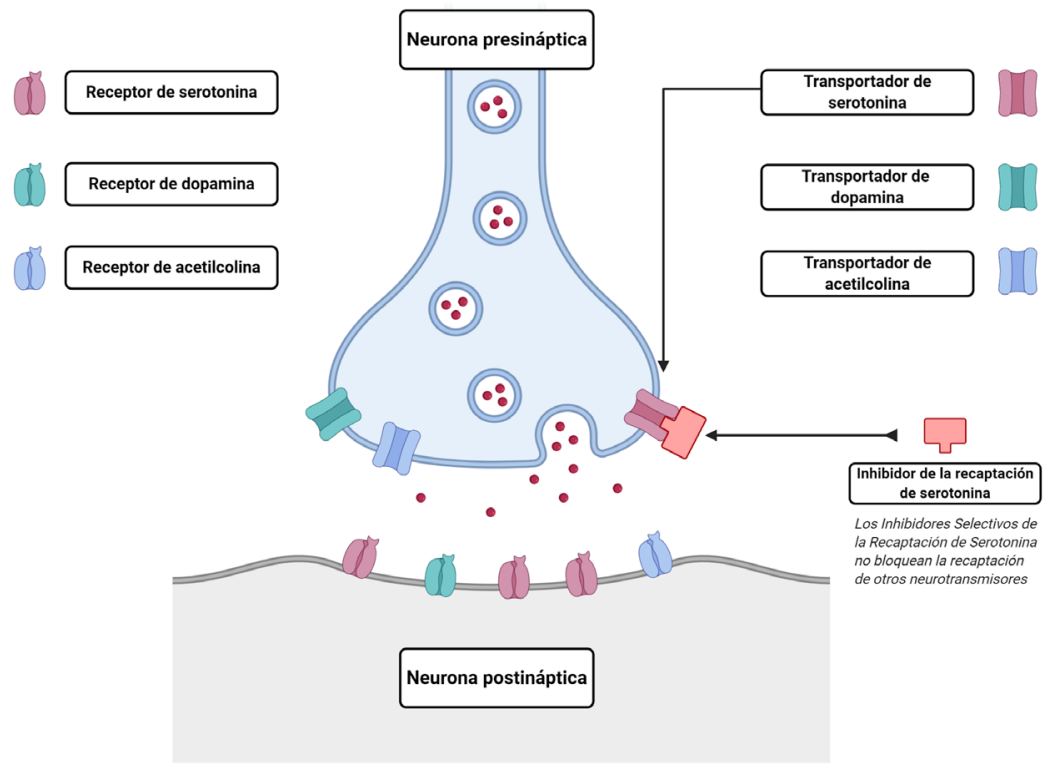

Por su parte, el modelo DED se fundamenta en la alteración de los mecanismos de neurotransmisión necesarios para llevar a cabo las funciones ejecutivas, 
"Disfunciones cognitivas en adultos mayores con depresión" Jorge Darío López Sánchez y Dora Elizabeth Granados Ramos

Vol. 22, Núm. 2, marzo-abril 2021

Revista Digital Universitaria

entre los que se encuentran la dopamina, la norepinefrina, la histamina, la acetilcolina y la serotonina, por lo que se propone que, en las personas con disfunción ejecutiva depresiva, la respuesta al tratamiento con IsRS será menor (McLennan y Mathias, 2010). Derivado de lo anterior, si se detectan a las personas con disfunción ejecutiva y sintomatología depresiva, es posible establecer un plan de tratamiento adecuado para cada caso, que incluya fármacos distintos a los ISRS para modificar las concentraciones de los demás neurotransmisores, y así se favorezca el funcionamiento ejecutivo y se disminuyan los síntomas depresivos. En este sentido, es importante que un equipo de psicólogos clínicos, neuropsicólogos y psiquiatras realicen una valoración exhaustiva e integral de las personas atendidas.

\section{Conclusiones}

Las complicaciones cognitivas comórbidas al trastorno depresivo en adultos mayores son numerosas. Se pueden observar desempeños bajos en tareas donde participan las funciones ejecutivas, la memoria, la atención y la velocidad de procesamiento. Dichas complicaciones deterioran aún más la calidad de vida del adulto mayor que padece depresión y, de la misma forma, dificultan su desenvolvimiento en actividades cotidianas, al modificar negativamente su autoconcepto y empeorar su sintomatología afectiva.

La evaluación y categorización de las dificultades cognitivas presentes en el trastorno depresivo en personas adultas mayores son herramientas que sirven de guía al profesional para establecer un diagnóstico diferencial entre el deterioro cognitivo comórbido y el trastorno afectivo, así como el que precede a una neuropatología. La identificación temprana de las complicaciones cognitivas permite a los especialistas en salud mental elaborar planes de tratamiento psicoterapéutico individualizados para mitigar los déficits que las personas adultas mayores presentan y establecer un tratamiento farmacológico más apropiado. Sin embargo, en México la prevalencia de enfermedades como obesidad, diabetes e hipertensión es elevada, y estas condiciones de salud también deterioran la función ejecutiva, por lo que son otros factores que considerar.

Es por esto que la valoración neuropsicológica es de suma importancia en el envejecimiento, ya que en los adultos mayores que presentan disfunción ejecutiva comórbida a la sintomatología depresiva, los ISRS pueden tener un efecto reducido, por lo que se sugiere analizar el tratamiento con distintos fármacos. El enfoque que ha demostrado mayor efectividad para tratar la depresión es la terapia cognitivo conductual enfocada a resolución de problemas, que favorece el funcionamiento ejecutivo debido a que, durante el proceso psicoterapéutico, la persona identifica situaciones problemáticas, y planea, desarrolla y lleva a cabo estrategias cognitivas y afectivas para afrontarlas, estimulando así, procesos cognitivos y habilidades que mejorarán su calidad de vida. Este enfoque terapéutico es más efectivo si se acompaña de una intervención farmacológica adecuada. 


\section{Referencias}

* Alexopoulos, G. S. (2001). The depression-executive dysfunction syndrome of late life: a specific target for D3 agonists? Am J Geriatr Psychiatry, 9(1), 22-29. Dol: https://www.doi.org/10.1097/00019442-200102000-00004

* American Psychological Association (APA). (2020). Depression. American Psychological Association. https://www.apa.org/topics/depression/

- Baune, B., Malhi, G., Morris, G., Outhred, T., Hamilton, A., Das, P., Basset, D., Berk, M., Boyce, P., Lyndon, B., Mulder, R., Parker, G. y Singh, A. (2018). Cognition in depression: Can we THINC-it better? Journal of Affective Disorders, 225, 559-562. Dol: https://www.doi.org/10.1016/j.jad.2017.08.080

* Bogner, H. R., Bruce, M. L., Reynolds III, C. F., Mulsant, B. H., Cary, M. S., Morales, K. y Alexopoulos, G. S. (2007). The effects of memory, attention, and executive dysfunction on outcomes of depression in a primary care intervention trial: the PROSPECT study. Int J Geriatr Psychiatry, 22, 922-929. Dol: https://doi.org/10.1002/ gps.1767

* Brown, P. J., Sneed, J. R., Rutherford, B. R., Devanand, D. P., y Roose, S. P. (2014). The nuances of cognition and depression in older adults: the need for a comprehensive assessment. Int J Geriatr Psychiatry, 29(5), 506-514. Dol: https://doi.org/10.1002/ gps.4033

* Crocco, E. A, Castro, K. C. y Loewenstein, D. A. (2010). How Late-Life Depression Affects Cognition: Neural Mechanisms. Current Psychiatry Reports, 12, 34-38. Dol: https://doi.org/10.1007/s11920-009-0081-2

* Donovan, N. J., Wu, Q., Rents, D. M., Sperling, R. A., Marshall, G. A. y Glymour, M. M. (2017). Loneliness, depression and cognitive function in older U.S. Adults. Int J Geriatr Psychiatry, 32 (5), 564-573. Dol: https://doi.org/10.1002/gps.4495

- Fischer, C., Schweizer, T. A., Atkins, J. H., Bozanovic, R., Norris, M., Herrmann, N., Nisenbaum, R. y Rourke, S. B. (2008). Neurocognitive profiles in older adults with and without major depression. Int J Geriatr Psychiatry, 23(8), 851-856. Dol: https:// doi.org/10.1002/gps.1994

* Hiemke, C. y Härtter, S. (2000). Pharmacokinetics of selective serotonine reuptake inhibitors. Pharmacology \& Therapeutics, 85(1), 11-28. Dol: https://doi.org/10.1016/ S0163-7258(99)00048-0

* McLennan, S. y Mathias, J. (2010). The depressión-executive dysfunction (DED) syndrome and response to antidepressants: a meta-analytic review. Int J Geriatr Psychiatry, 25(10), 933-944. Dol: https://doi.org/10.1002/gps.2431

* Pantzar, A., Atti, A. R., Fratiglioni, L., Fastbom, J., Bäckman, L., y Laukka, E. J. (2017). Cognitive performance in unipolar old-age depression: a longitudinal study. Int J Geriatr Psychiatry, 32(6), 675-684. Dol: https://doi.org/10.1002/gps.4510

* Ritchie, H. y Roser, M. (2018, January 20). Mental Health. Our World in Data. https:// ourworldindata.org/mental-health 
* Thomas, A. J. y O'Brien, J. T. (2008). Depression and cognition in older adults. Current Opinion in Psychiatry, 21(1), 8-13. Dol: https://doi.org/10.1097/ YCO.0b013e3282f2139b

* Weisenbach, S. L., Boore, L. A. y Kales, H. C. (2012). Depression and Cognitive Impairment in Older Adults. Current Psychiatry Reports, 14, 280-288. Dol: https:// doi.org/10.1007/s11920-012-0278-7

* World Health Organization: [who]. (2017, January 3). Depression and Other Common Mental Disorders. https://www.who.int/publications-detail/depression-globalhealth-estimates

* World Health Organization [wHo]. (2019, December 19). Mental Health. https:// www.who.int/health-topics/mental-health

\section{Material complementario}

Si el lector desea conocer más, los autores recomiendan los libros: El cerebro ejecutivo por Elkhonon Goldberg, Neuropsicología de la corteza prefrontal y las funciones ejecutivas por Javier Tirapu Ustárroz, y Envejencimiento cognitivo por Denise Park y Norbert Schwarz.

\section{Cómo CITAR ESTE ARTículo}

* López Sánchez, Jorge Darío y Granados Ramos, Dora Elizabeth. (2021, marzoabril). Disfunciones cognitivas en adultos mayores con depresión. Revista Digital Universitaria(RDU),22(2).Dol:http://doi.org/10.22201/cuaieed.16076079e.2021.22.2.2 